\title{
Association of Bacterial Endocarditis and Buccomaxillofacial Surgery: Review of Current Medical Literature
}

\author{
Éber Coelho Paraguassu ${ }^{1}$, Douglas Voss ${ }^{2}$, Salomão Barauna Alcolumbre ${ }^{3}$, Irlan Fernandes \\ Bacelar $^{4}$, Emilio Daniel Pacheco de Sousa ${ }^{5}$, Jose Thiers Carneiro Junior ${ }^{2}$
}

${ }^{1}$ Department of Odontological Sciences, GOE/UNIAVAN, Macapá, Amapá, Brazil, ${ }^{2}$ Department of Odontological Sciences, Universidade Federal do Pará, Belem, Pará, Brazil, ${ }^{3}$ Department of Cardiology, Hospital Cruz Azul-São Paulo, São Paulo, Brazil, ${ }^{4}$ Department of Cardiology, Hospital de Emergencia Osvaldo Cruz, Macapá, Amapá, Brazil, ${ }^{5}$ Department of Health Sciences, Hospital de Clínicas Dr. Alberto Lima, Macapá, Amapá, Brazil

\begin{abstract}
This review article seeks to provide additional information on bacterial endocarditis and Buccomaxillofacial Surgery and Traumatology procedures, as well as the main microorganisms involved in this feat, risk factors, and conditions and antibiotic prophylaxis. A bibliographic review was carried out, where scientific articles were obtained from the PubMed, Cochrane, Virtual Health Library and Scielo databases. Studies were conducted from 2000 to 2018 and included studies from the period 2013 to 2018 , according to the descriptors: Bacterial Endocarditis-Prevalence-Prophylaxis-Bacterial, as well as the association of the terms, searched in English, Spanish and Portuguese. A total of 189 studies were carried out to carry out the research and included 13 articles. Data were analyzed from descriptive statistics. Through information exported in these works, it can be concluded that the best way to prevent an infective endocarditis is to know the patient by means of a well-made anamnesis, where the patient's health history is well evaluated and thus to identify some risk, and antibiotic prophylaxis is recommended for all dental patients of imminent risk, as well as more invasive surgery and buccomaxillofacial procedures.
\end{abstract}

Key Words: Bacterial endocarditis, Prevalence, Antibioticoprofilaxia, Oral surgery

\section{Introduction}

Bacterial endocarditis (EB), initiated by bacteremia is an infection that involves especially the valvar endocardium, but it affects structures such as communication endocardium ventricular septal defects and valvular prostheses. It is a disease rarely capable of causing serious sequelae and even death if not treated quickly [1]. Although there are still doubts about the etiology of bacterial endocarditis after dental treatments, bacteremias spontaneous transients are the most likely causes [2].

Periodontal probing, surgeries or even stringent dental brushing, for example, may lead to the introduction and consequent dissemination of bacteria belonging to the microbiota of the oral cavity in the current circulatory system. This condition is called bacteremia transient, which in normal organisms is quickly eliminated by host defenses [3]. Every structure in basic life support that we have and several technologies for diagnosis, today do not seem to be enough, since mortality rates due to infective endocarditis reach about $25 \%$ [3].

Nowadays, discussions about Surgery and Traumatology Buccomaxillofacial (STBMF) and endocarditis infectious disease due to the oral microbiota involved in these surgical procedures, seek to clarify the type of treatment available for the disease, and mainly, the maneuvers to be taken aiming at its prevention [4].

In response to major doubts and challenges lived in the past, which is perpetuated until the today, the literature review study has objectives, to outline additional information on microbial etiology of bacterial endocarditis in STBMF procedures, main microorganisms involved, factors and conditions of as well as antibiotic prophylaxis and its prevention.

\section{Material and Methods}

A literature review was performed, where scientific articles were obtained from the PubMed, Cochrane, Virtual Health Library and Scielo databases. Studies from 2000 to 2018 and included studies from 2013 to 2018, according to the keywords: Bacterial Endocarditis-Prevalence-ProphylaxisBacterial, as well as the association of the searched terms in English, Spanish and Portuguese languages. Totaling 189 studies to perform the research and included 13 articles that had as inclusion criteria the high impact factor where it was published and indexing in Sucupira platform (Index Qualis). Data were analyzed using descriptive statistics.

\section{Literature Revision}

At the moment a therapeutic manipulation in a patient, pathogenic micro-organisms spontaneously or inoculated during the procedure, lead to transient bacteremias, which result in systemic alterations, including endocarditis infectious (EI) [2].

Transient bacteremias may occur, for a turn, even in the absence of procedures dental hygiene or periodontal disease, as well as infections periapical, periodontal and buccal mucosa. Therefore, many authors argue that the estimated risk of IE related to dental procedures sporadic is low [4-7].

Among the clinical characteristics observed in patients with IE are high fever, weight loss, lethargy, difficulty breathing, heart murmur new immunological phenomena, manifestations such as Osler's nodules and the lesions of Janeway. The main complications include including sepsis, stroke, and heart failure due to valve dysfunction $[4,7,8]$.

The clinical judgment from the history and the clinical examination of the patient is essential for the diagnosis of

Corresponding author: Dr. Éber Coelho Paraguassu, Department of Odontological Sciences, GOE/UNIAVAN, Macapá, Amapá, Brazil, E-mail: paraguassu84@yahoo.com.br 
endocarditis, confirmed with the complementary examinations and findings histological and microbiological studies of blood cultures positive for EI specific bacteria [4]. Among the complementary examinations are the transthoracic echocardiography and echocardiography transesophageal. The first allows bacterial vegetations, abscesses, valve lesions, peripheral impairment, as well as providing valuable information about the ventricular function. On the other hand, transesophageal echocardiography especially in the presence of prosthetic valves cardiac or intracardiac devices.

Gram-positive cocci are the microorganisms in endocarditis infectious diseases, as well as those responsible for their cause. As representatives of this group, Streptococcus, being the most prevalent species of group viridans, Staphylococcus, being the most frequently involved S. aureus, Enterococcus faecalis and microorganisms of the HACEK group. Fungi are also cited in the literature as agents etiological [7-10].

For prevention of infective endocarditis, it is of fundamental importance that an anamnesis well-done before any treatment invasive dentistry and the dialogue between dentists and cardiologists in the case of patients with to determine the need for antibiotic prophylaxis [2]. In addition, patients who sign and symptoms suggestive of IE after dental procedures should be referred for treatment as soon as possible [7].

Antibiotic prophylaxis in cases of fracture of jaws is a basic need for several decades. It is worth noting that although the treatment of infective endocarditis is not the competence of the surgeon bucomaxillofacial, this does not diminish its responsibility for such status as an agent of health. The success of endocarditis treatment infectivity varies according to its severity and virulence of the microorganisms involved and such as early diagnosis and initiation of treatment, which can be medicated, through (antibiotic therapy) or surgical treatment by means of (valve replacement affected) [10].

Quantity, intensity, prevalence, and duration of invasive procedures determine the bacteremia [10]. In this way, the staging of the infective endocarditis becomes proportionally depending on the incidence, as well as the intensity of bacteremia [11], which can be classified as acute when there are pathogens of great virulence, generating an exuberant clinical and early complications; and subacute, when the pathogens are of lower virulence, having a slow evolution [2].

The indiscriminate prescription of antibiotics, besides its adverse effects, favors resistance bacteria, mainly of microorganisms associated with the etiopathology of infective endocarditis. A total of $2 \%$ of oral and maxillofacial surgeries result in infection of the surgical site, it is to postoperative follow-up. It has been realized in the literature that Intraabdominal surgeries are probably the most wounds associated with infection. individuals submitted to surgeries of the complex those who also have one of the infections [5].

In cases of maxillofacial trauma, some factors should be evaluated against the incidence of infection and selection of antibiotic prophylaxis. The location of the trauma is extreme factor importance. Thus, the invasion of microorganisms in the site of fractures in the bones of the face of the closed type, such as condyle and mandibular branches, as well as such as
Le Fort I and III fractures, occurs in a different way of the exposed fractures, with direct communication between the cavity and/or skin surface $[8,11]$.

No study was found that assessed/reported the degree of invasion by microorganisms in the fractures consolidated or in process of bone healing in the bones of the face, the in spite of the importance of these studies, since fractures classified as closed presented low infection rates when compared to exposed fractures $[7,10]$.

The incidence of bacterial endocarditis showed a significant difference in relation to the type of access for intraoral or extraoral fractures [11]. this is another factor to be desired in the literature. The use of antimicrobial agents is crucial importance in buccomaxilofacil surgery, once that such procedures involve the nasal cavity, mouth, and sinus, where there is a vast flora of microorganisms with high virulence factor agents involved in the prevention and treatment of possible infections [10].

According to the literature findings, the benefit of antibiotic prophylaxis in the prevention of mandibular fractures. However, the studies in question do not clarify the duration of use of such based pharmaceuticals [5].

\section{Discussion}

Bacteremia may be secondary to dental procedures, which may occur in greater or lesser proportion. About infective endocarditis (IE) due to bucomaxillofacial procedures, the literature has controversial and controversial in some points and concisely in other respects, showing the divergence information on the etiology and treatment $[5,12]$.

The literature has shown consistently that the patient's previous contact with pathogenic microorganisms as well as maxillofacial injuries, iatrogenic association with patient hygiene, are factors the evolution of the infection. More of 250 species of microorganisms form the resident microbiota of the oral cavity, developing different pathological conditions in moments that the patient's immunity seems to fail $[7,12]$.

Bacteremia is considered a step important for the onset of IE and especially induced by invasive dental treatments, including dental extraction, periodontal surgery, scaling and polishing, as well as trauma maxillofacial. In addition, it is consensus in the literature that routine oral hygiene procedures, such as tooth brushing, flossing and chewing may also allow the induction of bacteremia $[2,10]$. Thus, invasive dental treatments are the main cause of EI, becoming an issue controversial in the literature and concise concerning fractures the maxillofacial region [2].

However, according to the same authors, transient bacteremias do not have the qualitative, much less quantitative for developing colonization and infection pictures, even in unfavorable cardiac patients. Much dental works for which antibiotic prophylaxis is recommended is those capable of inducing bacteremia, which would be critical in patients with cardiac conditions predisposing [2]. However, $50 \%$ of IE cases can develop in patients without known cardiac valve injury $[7,10]$. 
Studies show that 30 to 60 minutes after oral surgery a small percentage of blood culture is positive for Streptococcus viridans, but without clinical repercussion. Besides that, the majority of patients with endocarditis did not by tissue injury of the maxillofacial region in the weeks before the onset of symptoms. Thus, the risk of endocarditis under a buccodent's surgical procedure is less than $15 \%$ and prophylaxis would prevent only a small number of cases, this is considered to be $100 \%$ effective [5].

In the same way, the literature reports that patients are at higher cumulative risk of IE daily because of routine activities than of surgical procedures oral diseases. Transient bacteremia is secondary to chewing and brushing result in 5370 minutes of bacteremia over a period of one month in comparison of 6-30 minutes resulting from a single tooth extraction [12,13].

Therefore, the focus has caused by dental procedures for the bacteremias generated by occurrences of daily life, cumulative process. But that does not mean say that the closed STBMF procedures are risk-free. The risk exists, but the occurrence is probably minimal to be demonstrated statistically in samples populations. In addition, the parameters that contribute most to the risk of infective endocarditis are still uncertain because there is no convincing evidence or evidence $[11,13]$. Thus, good oral hygiene and eradication of dental diseases are the most effective preventive methods to reduce daily bacteremias $[5,10,13]$.

Taking into account the different cardiopathies and their close relationship with EB, the American Heart Association (AHA) classified as high, moderate and low-risk factors and has been making constant updates on its prophylactic protocol, which occurred in 1955, 1965, 1972, 1977, 1984, 1990, 1997, 2007 and, more recently, in 2017. Until 2007, it was recommended to carry out antibiotic prophylaxis on patients with high and moderate factors risk [6].

However, from the guidelines in 2007, prophylaxis for endocarditis infectious disease is indicated for patients with cardiac conditions considered to be at high risk [1-13]. These recommendations are based on several studies retrospectives suggesting greater morbidity in these groups of patients $[6,7]$.

In the update on June 2017, the American College of Cardiology/American Heart Association (ACC/AHA) included patients with transcatheter prosthetic valves and patients with prosthetic material used for valve repair to those in the high-risk category that require prophylaxis. These additional indications were updated after recent observational studies, noting the high risk of IE and high risk of patients in these subgroups. The next guideline publication is not expected before 2018 [7].

Such guidelines have caused many controversies in the current literature due to the conflict of recommendations with the conduct in use by the majority of professionals, in addition to confronting different realities between the US and Brazil, reflected in the decision on the conduct to be adopted [5].

The AHA recommendations, published in 2007, did not change the suggested prophylactic regimens in 1997, whether in relation to antibiotics, dosage or at the time of administration of the drugs. The antibiotic prophylaxis should be performed from 2 hours prior to the dental procedure, is the ideal time from 30 to 60 minutes in advance. However, the prophylactic dose can be administered up to 2 hours after the procedure, but only in the case that the patient inadvertently did not use the medication.

Important aspects related to the use of antibiotics are the effects that such drugs can generate and concern for bacterial resistance to antibiotics in clinical practice $[6,10]$. The research suggested that the risk of fatal anaphylactic penicillin is considerably greater than that of contracting infective endocarditis [11]. However, these AHA itself reported having no knowledge of fatal anaphylaxis associated with antibiotics employed in the prophylaxis protocol of endocarditis in the United States during the last 50 years [10].

All of these guidelines were based on and non-evidencebased effectiveness of pre-procedure antibiotics for EI prevention has never been proven in a double randomized controlled trial. A bank of a systematic review of the Cochrane antibiotic prophylaxis in dentistry concluded that there is no evidence to determine whether prophylaxis antibiotic prior to STBMF procedures is effective or ineffective [7].

Meanwhile, the best approach Bucomaxillofacial Surgeon and Traumatologist can follow the AHA: The 2007 guidelines and the update of AHA/ACC 2017 in prescribing antibiotic prophylaxis for high risk patients and emphasize the importance of optimizing health to reduce the incidence of bacteremia by daily activities such as chewing, brushing teeth and flossing [7].

The most common causative organisms of Reported in Japan are oral streptococci, followed by staphylococci, indicating that prevention should be considered by collaborative discussions between dentists and cardiologists. On the other hand, recent findings have found that knowledge among dentists on the guidelines for IE prevention is insufficient, while cardiologists generally do not are aware of the dental treatments performed and if prophylactic antibiotics are generally used by dentists Filling the gap in practice clinic between dentists and cardiologists is considered imperative $[2,12]$.

Literature brings the latest and most comprehensive systematic review and meta-analysis of all studies available from 1960 to 2016 on prophylaxis antibiotics for infective endocarditis, concluding that the evidence is limited to define benefits and risks and not reaching a conclusion on the efficacy of antibiotic prophylaxis [13].

Being of consensus in the literature the patients with previous IE, cyanotic congenital heart disease, prosthetic valves and transplant recipients that developed valvular heart disease the highest risk group for endocarditis infection with surgical procedures $[7,13]$.

\section{Conclusion}

Through the scientific databases studied in this study, it becomes evident that changes in concepts, as well as the doubts that still perpetuate in relation to antibiotic prophylaxis in surgery and traumatology procedures bucomaxillofacial surgery, as well as in dentistry, However, it is the consensus of all authors studied in this study that antibiotic therapy is an 
indispensable step in the surgical procedure. From the information, it is possible to conclude that the best way to if preventing infective endocarditis is known the patient through a careful anamnesis, where the whole dental medical history is known of the patient so that it can be identified, individualized, risky and patients with unnecessary exposure to antibiotics. Antibiotic prophylaxis is recommended for all the eminent risk dental patients, as well as the surgery and bucomaxillofacial traumatology.

\section{References}

1. Habib G, Lancellotti P, Antunes MJ, Bongiorni MG, Casalta JP, et al. 2015 ESC Guidelines for the management of infective endocarditis: The task force for the management of infective endocarditis of the European Society of Cardiology (ESC). Endorsed by: European Association for Cardio-Thoracic Surgery (EACTS), the European Association of Nuclear Medicine (EANM). European Heart Journal. 2015; 36: 3075-3128.

2. Nomura R, Kokomoto K, Ohara T, Nakatani S, Ooshima T, et al. Current knowledge among Japanese experienced general dentists regarding prevention of infective endocarditis. Odontology. 2018; 106: $297-305$

3. Kaura A, Dworakowska D, Dworakowski R. Infective endocarditis-Cinderella in cardiology. Kardiologia Polska. 2017; 75: 965-974

4. Suda KJ, Henschel H, Patel U, Fitzpatrick MA, Evans CT. Use of Antibiotic prophylaxis for tooth extractions, dental implants, and periodontal surgical procedures. Open Forum Infectious Diseases. 2017; 5: ofx250.
5. Robinson AN, Tambyah PA. Infective endocarditis-An update for dental surgeons. Singapore Dental Journal. 2017; 38: 2-7.

6. Pericas JM, Corredoira JM. Miro Colorectal adenomas. The New England Journal of Medicine. 2016; 375: 387-390.

7. Chee QZ, Tan YQB, Ngiam JN, Win MTM, Shen X, et al. The Sharpen clinical risk score predicts mortality in patients with infective endocarditis: an 11-year study. International Journal of Cardiology. 2015; 191: 273-276.

8. Pang PYK, Sin YK, Lim CH, Tan TE, Lim SL, et al. Surgical management of infective endocarditis: an analysis of early and late outcomes. European Journal of Cardio-Thoracic Surgery. 2015; 47 : 826-832.

9. Liew WK, Tan TH, Wong KY. Infective endocarditis in childhood: a seven-year experience. Singapore Medical Journal. 2004; 45: 525-529.

10. Chong E, Poh KK, Shen L, Yeh IB, Chai P. Infective endocarditis secondary to intravenous Subutex abuse. Singapore Medical Journal. 2009; 50: 34-42.

11. Allegranzi B, Bagheri Nejad S, Combescure C, Graafmans $\mathrm{W}$, Attar $\mathrm{H}$, et al. Burden of endemic health-care-associated infection in developing countries: systematic review and meta-analysis. Lancet. 2011; 377: 228-241.

12. Blair JMA, Webber MA, Baylay AJ, Ogbolu DO, Piddock LJV. Molecular mechanisms of antibiotic resistance. Nature Reviews Microbiology. 2015; 13: 42-51.

13. Éber Coelho Paraguassu, Anneli Celis Mercedes de Cardenas, Marina Nolli Bittencourt, Ana Rita Pinheiro Barcessat, Paulo Fabricio Ramos. Quality of life and satisfaction of users of total tissue-supported and implant-supported prostheses in the municipality of macapá, Brazil. International Journal of Development Research. 2019; 9: 26007-26011. 